\title{
THE FOURTH ALL INDIA PEDIATRIC CONFERENCE
}

\section{POONA}

\author{
February 27-March I, I953.
}

The Conference of Pediatricians in India will be held in Poona from February 27-March I, 1953.

All members of the Indian Pediatric Society are cordially invited to attend the Conference and participate in its proceedings by contributing scientific papers and otherwise.

Those, who wish to attend, will please communicate at once with the Secretary, Indian Pediatric Society. Request for accommodation should be addressed to Dr. Chandrachud, Saraswati Vilas, Laxmi Road, Poona 2. 